\title{
Coulometric titration at low temperatures-nonstoichiometric silver selenide
}

\author{
Gesa Beck*, Jürgen Janek \\ Physikalisch-Chemisches Institut, Justus-Liebig-Universität Gießen, Heinrich-Buff-Ring 58, D-35392 Giessen, Germany
}

\begin{abstract}
A modified coulometric titration technique is described for the investigation of nonstoichiometric phases at low temperatures. It allows to obtain titration curves at temperatures where the conventional coulometric titration technique fails because of too small chemical diffusion coefficients of the mobile component. This method for indirect coulometric titration is applied to silver selenide between -100 and $100{ }^{\circ} \mathrm{C}$. The titration curves are analyzed on the basis of a defect chemical model and provide thermodynamic data of the $\alpha$-silver selenide phase. The boundaries of the homogeneous phase field are determined.
\end{abstract}

\section{Introduction}

Currently the nonstoichiometric low temperature $\alpha$-phase of $\mathrm{Ag}_{2}+{ }_{\delta} \mathrm{Se}$ (stable at $T<132{ }^{\circ} \mathrm{C}$ ) is subject to strong interest in its magnetoresistive (MR) properties [1-3] at ambient and low temperatures. It is now well known that a silver excess is required in order to obtain silver selenide samples with unusually large MR effects and it is also known that the microstructure of silver-rich $\alpha-\mathrm{Ag}_{2}+\delta$ Se plays a crucial role for the MR effect. It has been shown that a two-phase mixture of $\alpha-\mathrm{Ag}_{2}+\delta$ Se and $\mathrm{Ag}$ is required in order to observe a MR effect which is different from the ordinary magnetoresistance effect (OMR) [3]. In this context, the homogeneity range is of primary importance for the synthesis of well-defined specimens and the study of the MR effect in single-phase material.

A phase transformation from the cubic high temperature phase $\left(\beta-\mathrm{Ag}_{2}+\delta \mathrm{Se}\right)$, which is a prominent material in solid state electrochemistry, to the low temperature phase ( $\alpha$ $\mathrm{Ag}_{2+\delta} \mathrm{Se}$ ) occurs at $T=132{ }^{\circ} \mathrm{C}$. The low-temperature $\alpha$ phase exists only in a very small range of homogeneity which becomes narrower with decreasing temperature, as

\footnotetext{
* Corresponding author. Tel.: +49-641-9934501; fax: +49-641-9934509 .

E-mail address: gesa.beck@phys.chemie.uni-giessen.de (G. Beck).
}

indicated schematically in Fig. 1 and as usual for nonstoichiometric compounds. Up to now, the phase diagram (non-stoichometric data) of the system Ag-Se is reported only for temperatures between 75 and $190{ }^{\circ} \mathrm{C}[4,5]$.

In the present paper, we report the experimental determination of the phase field of the low temperature $\alpha$-phase from 100 down to $-100{ }^{\circ} \mathrm{C}$ by means of a modified coulometric titration technique [6]. From the thermodynamic interpretation of the titration curves, we are able to extend the phase diagram and to determine thermodynamic data for point defects in $\alpha$-silver selenide at low temperatures.

\section{Experimental}

Homogeneous $\beta$-silver selenide has been grown in a glass capillary by one-dimensional growth from the elements at $T=240{ }^{\circ} \mathrm{C}$. On one side of the capillary a rod of silver metal (purity 99.995\%) was placed. On the other side selenium was filled into the capillary and melted. Silver reacted with selenium and a coarse-grained $\beta$-silver selenide crystal grew into the capillary with a growth rate of approximately $10 \mathrm{~mm} /$ day. By cooling slowly down to temperatures below $132{ }^{\circ} \mathrm{C}$, the $\beta \rightarrow \alpha$-transformation takes place. Due to the significant density increase of about $2 \%$ $\left[V_{\mathrm{m}}\left(\alpha-\mathrm{Ag}_{2} \mathrm{Se}\right)<V_{\mathrm{m}}\left(\beta-\mathrm{Ag}_{2} \mathrm{Se}\right)\right]$ during the transformation 


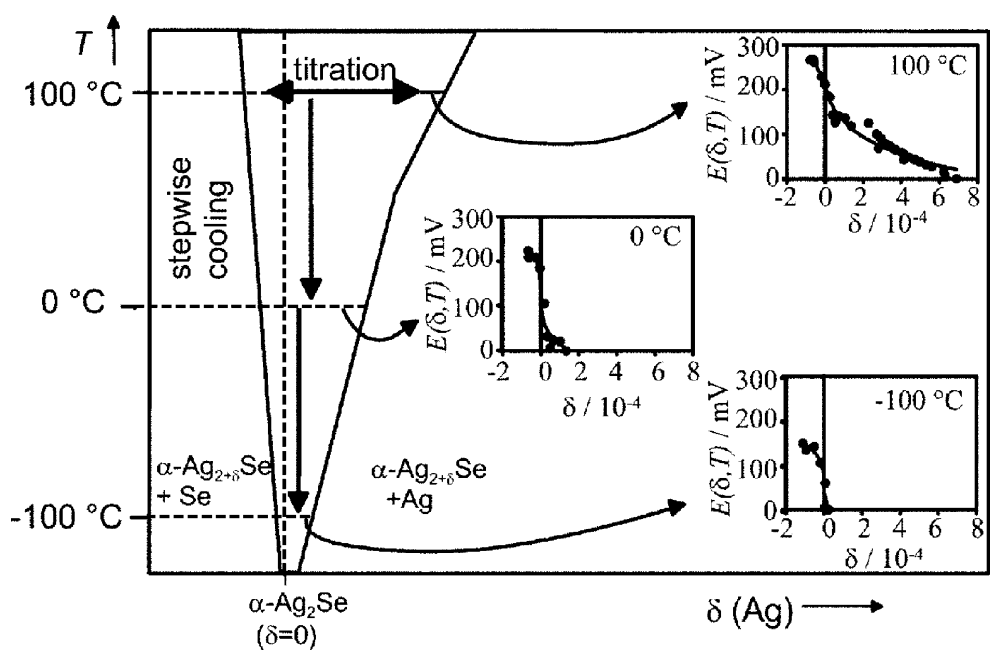

Fig. 1. Schematic part of the phase diagram of the system silver-selenium at low temperatures. Insets: Diagrams of the electromotive force $E(\delta, T)$ versus the metal excess $\delta$ and fitted coulometric titration curves at 100,0 and $-100{ }^{\circ} \mathrm{C}$ (experimental results from this work).

from the $\beta$-phase, dislocations and grain boundaries are created in the $\alpha$-phase and lead to a material with a high concentration of non-equilibrium defects. In order to heal out these defects at least partially and to obtain a material with defined silver activity, the crystal was subsequently equilibrated with bulk silver metal for several weeks at 100 ${ }^{\circ} \mathrm{C}$. This long equilibration period is necessary since the chemical diffusion coefficient of silver in the $\alpha$-phase is relatively low compared to that of the $\beta$-phase (e.g. $\tilde{D}_{\mathrm{Ag}}=0.6 \times 10^{-5} \mathrm{~cm}^{2} \mathrm{~s}^{-1}$ at $100{ }^{\circ} \mathrm{C}$ and $\delta=0$ [7]). Finally, a polycrystalline product with coarse grains and with a silver activity $a_{\mathrm{Ag}}=1$ at $100{ }^{\circ} \mathrm{C}$ was obtained.

With this starting material, we constructed an electrochemical cell for coulometric titration which is depicted in Fig. 2. We used $\mathrm{RbAg}_{4} \mathrm{I}_{5}$ with a high silver ion conductivity even at low temperatures and negligible electronic conductivity as solid electrolyte. The use of a two-phase electrode $\mathrm{RbAg}_{4} \mathrm{I}_{5}+\mathrm{Ag}$ increases the exchange current density of the electrode interface and, thus, improves the stability of the electromotive force $E(\delta, T)$. Depending on the direction of the electric current across the resulting cell:

\section{$\mathrm{Ag} / \mathrm{RbAg}_{4} \mathrm{I}_{5} / \alpha-\mathrm{Ag}_{2} \mathrm{Se} / \mathrm{Pt}$}

silver is added or removed from the $\alpha-\mathrm{Ag}_{2} \mathrm{Se}$ specimen and the deviation $\delta$ from stoichiometry is changed with high precision. These processes are illustrated in Fig. 2.

Silver addition and removal were always performed at $100{ }^{\circ} \mathrm{C}$, where silver selenide shows sufficiently high silver mobility. After adjusting a definite deviation $\delta$ from stoichiometry, we cooled the cell in well-defined steps down to $80,50,20,0,-30$ and $-100{ }^{\circ} \mathrm{C}$ (see Fig. 1). At each temperature we measured the electromotive force $E(\delta, T)$ after equilibration. By doing so, we firstly collected data of the type $E$ vs. $T$ for constant composition [ $E(\delta=$ const., $T)]$. Secondly, several data series were then used to construct isothermal data series of the type $E(\delta, T=$ const. $)$.
The reproducibility of the measurement was scrutinized by measuring $E(\delta=$ const., $T)$ again during heating up the cell. These data were compared with those from

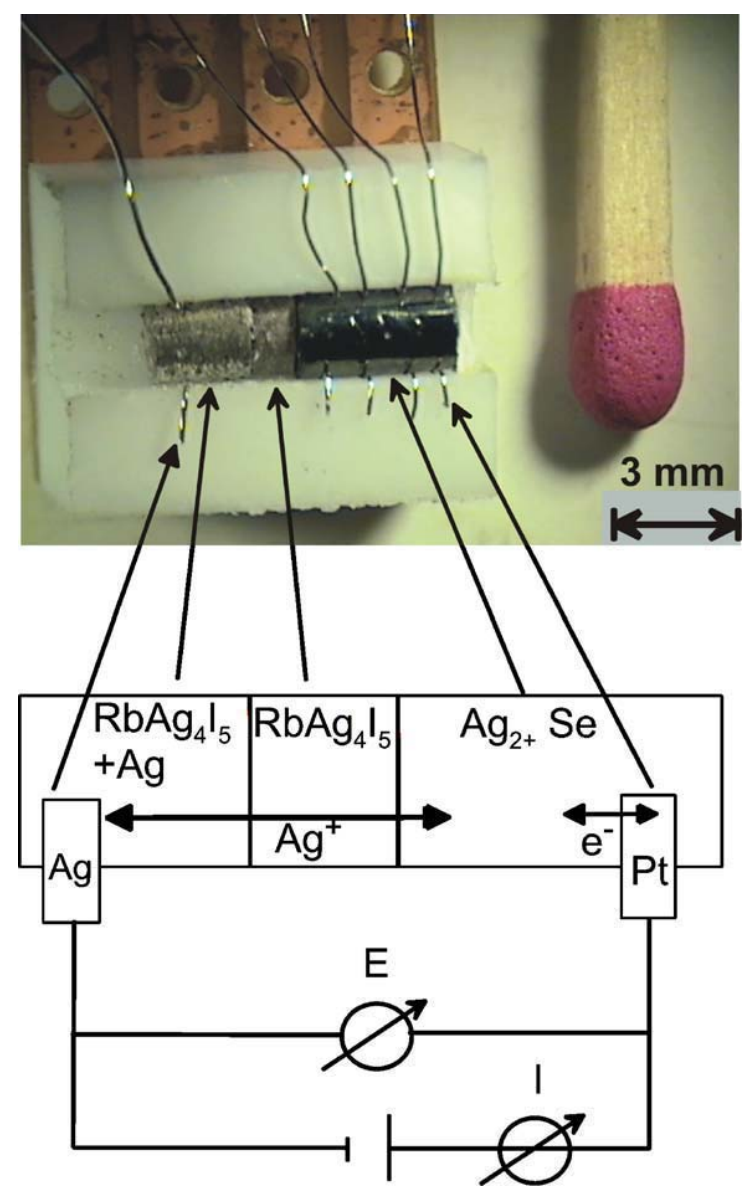

Fig. 2. Bottom: Electric circuit for coulometric titration of silver selenide. Top: Photograph of the miniaturized electrochemical cell for coulometric titration. This cell is constructed such that is can directly be used within a magnet for MR effect measurements. 
the cooling sequence. In addition, we changed $\delta$ firstly in the direction of high silver activity and then back in direction of low silver activity. At different values of $\delta$, we measured the $E(\delta=$ const., $T)$ data. This temperature and composition cycling procedure guarantees the reproducibility of the measurements.

The temperature of the sample was controlled by a flux of nitrogen gas (obtained from liquid nitrogen), as a part of a sample holder which can also be used for the magnetotransport studies. It has been measured by a Ni-CrNi-thermocouple near the sample. The thermocouple element was combined with an Oxford-ITC4 which controlled the power of the gas heater. With this method we were able obtain a constant temperature of the sample within a deviation of $\pm 0.1{ }^{\circ} \mathrm{C}$. The galvanostatic titration was realized with a Jaissle potentiostat in a galvanostatic mode. A Keithley voltmeter (DMM2001) was used for the precise measurement of all voltages.

\section{Experimental results and discussion}

In the insets of Fig. 1, the indirectly determined titration curves at 100, 0 and $-100{ }^{\circ} \mathrm{C}$ are plotted as examples (fits to experimental data). The experimental data are marked by filled symbols. The curves are fitted to the data by the use of Eq. (3). by:

The change of the silver excess $\Delta \delta$ in $\mathrm{Ag}_{2}+{ }_{\delta} \mathrm{Se}$ is given

$\Delta \delta=\frac{V_{\mathrm{m}}}{F \cdot V_{\text {sample }}} \int_{\Delta t} I_{\mathrm{titr}} \cdot \mathrm{d} t=\frac{V_{\mathrm{m}}}{F \cdot V_{\text {sample }}} I_{\mathrm{titr}} \cdot \Delta t$

where $I_{\text {titr }}$ is the constant titration current, $\Delta t$ denotes the titration time, $V_{\mathrm{m}}\left(=37.48 \mathrm{~cm}^{3} \mathrm{~mol}^{-1}\right)$ represents the molar volume of silver selenide, $V_{\text {sample }}$ represents the volume of the sample and $F$ is the Faraday constant.

The electromotive force $E(\delta, T)$ of the cell (equilibrium) is:

$E(\delta, T)=\frac{\left(\mu_{\mathrm{Ag}}^{0}-\mu_{\mathrm{Ag}}\right)}{F}=-\frac{R \cdot T}{F} \ln a_{\mathrm{Ag}}$

with $\mu_{\mathrm{Ag}}^{0}, \mu_{\mathrm{Ag}}$ and $a_{\mathrm{Ag}}$ denoting the chemical potential of pure silver metal as reference, the chemical potential of silver metal in silver selenide and the silver activity in silver selenide, respectively. The relation between $E(\delta, T)$ and the silver excess $\Delta \delta$ follows from the defect thermodynamics of $\alpha-\mathrm{Ag}_{2}+\delta$ Se and is given by [8]:

$$
\begin{aligned}
E(\delta, T)= & E^{\#}(T)-\frac{R \cdot T}{F}\left[\operatorname{arsinh}\left(\frac{\Delta \delta-\Delta \delta\left(E^{\#}\right)}{2 \cdot K_{\mathrm{F}}^{1 / 2}}\right)\right. \\
& \left.+\operatorname{arsinh}\left(\frac{\Delta \delta-\Delta \delta\left(E^{\#}\right)}{2 \cdot K_{\mathrm{e}}^{1 / 2}}\right)\right] .
\end{aligned}
$$

Here $T$ is the temperature in Kelvin, $R$ the gas constant, $K_{\mathrm{F}}$ the Frenkel constant, $K_{\mathrm{e}}$ the equilibrium constant for electron-hole formation and $E^{\#}(T)$ the electromotive force at the stoichiometric point. Fitting the measured data points $E(\delta, T)$ versus $\Delta \delta$ to Eq. (3) results in values of $K_{\mathrm{F}}, K_{\mathrm{e}}$ and $E^{\#}(T)$ as summarized in Table 1 and $\Delta \delta\left(E^{\#}\right)$. The value of $\Delta \delta\left(E^{\#}\right)$ represents the shift of the titration curve along the $\delta$ axis, which is required to fix the inflection point at $\delta=0$. From $\Delta \delta$ and $\Delta \delta\left(E^{\#}\right)$, we determine absolute values of $\delta(E)$ (with: $\delta(E)=\Delta \delta-\Delta \delta\left(E^{\#}\right)$ ), relative to the inflection point of the curve (stoichiometric point) with $\delta=0$. With these we can construct the diagram of $E(\delta, T)$ versus $\delta$. The titration curve contains the information about the phase width for the given temperature. At $E(\delta, T)=0$, silver selenide is in equilibrium with silver $\left(a_{\mathrm{Ag}}=1\right)$. We chose the equilibrium with selenium at an activity $a_{\mathrm{Se}}=1$ as the opposite phase boundary in Fig. 3a and b. This isoactivity line is calculated from the thermodynamic decomposition voltage $E_{\mathrm{d}}(T)$ or the standard free enthalpy of formation of $\mathrm{Ag}_{2} \mathrm{Se}$ as:

$$
\begin{aligned}
E_{\mathrm{d}}\left(a_{\mathrm{Se}}\right. & =1, T)=-\frac{R \cdot T}{F} \ln \left[a_{\mathrm{Ag}}\left(a_{\mathrm{Se}}=1\right)\right] \\
& =-\frac{\Delta_{\mathrm{r}} G^{\bigcirc}(T)}{2 F} .
\end{aligned}
$$

With the points $\delta\left(a_{\mathrm{Ag}}=1\right)$ and $\delta\left(a_{\mathrm{Se}}=1\right)$ at different temperatures we constructed the boundaries of the phase field in the phase diagram in Fig. $3 \mathrm{a}$ and $\mathrm{b}$. It has to be noted that the phase field becomes wider for decreasing temperatures at $a_{\mathrm{Se}}=1$. The usual case is that the phase field becomes narrower on both sides with decreasing temperature. This narrowing depends on the details of the interaction between silver and selenium, which is reflected in the thermodynamic parameters of the silver-selenium solid solution. Only in unusual cases of a lower critical temper-

Table 1

Data obtained from coulometric titration of $\alpha-\mathrm{Ag}_{2}+{ }_{\delta} \mathrm{Se}$

\begin{tabular}{llllll}
\hline$T /^{\circ} \mathrm{C}$ & $\delta\left(a_{\mathrm{Ag}}=1\right)$ & $\delta\left(a_{\mathrm{Se}}=1\right)$ & $E_{\mathrm{d}}(T) / \mathrm{mV}$ & $E^{\#}(T) / \mathrm{mV}$ & $K_{\mathrm{F}}$ \\
\hline 100 & $7.86 \times 10^{-4}$ & $-3.5 \times 10^{-5}$ & 270.5 & 194.8 & $1.75 \times 10^{-11}$ \\
80 & $4.32 \times 10^{-4}$ & $-4.0 \times 10^{-5}$ & 268.0 & 183.6 & $5.76 \times 10^{-12}$ \\
50 & $1.75 \times 10^{-4}$ & $-5.0 \times 10^{-5}$ & 264.3 & 158.2 & $5.31 \times 10^{-8}$ \\
20 & $0.79 \times 10^{-4}$ & $-8.0 \times 10^{-5}$ & 260.7 & 129.7 & $7.15 \times 10^{-12}$ \\
0 & $0.55 \times 10^{-4}$ & $-1.2 \times 10^{-4}$ & 258.3 & 112.7 & $1.37 \times 10^{-12}$ \\
-30 & $0.38 \times 10^{-4}$ & $-1.4 \times 10^{-4}$ & 254.8 & 103.3 & $8.93 \times 10^{-13}$ \\
-100 & $0.33 \times 10^{-4}$ & $-3.20 \times 10^{-3}$ & 246.7 & 60.4 & $2.15 \times 10^{-13}$ \\
\hline
\end{tabular}




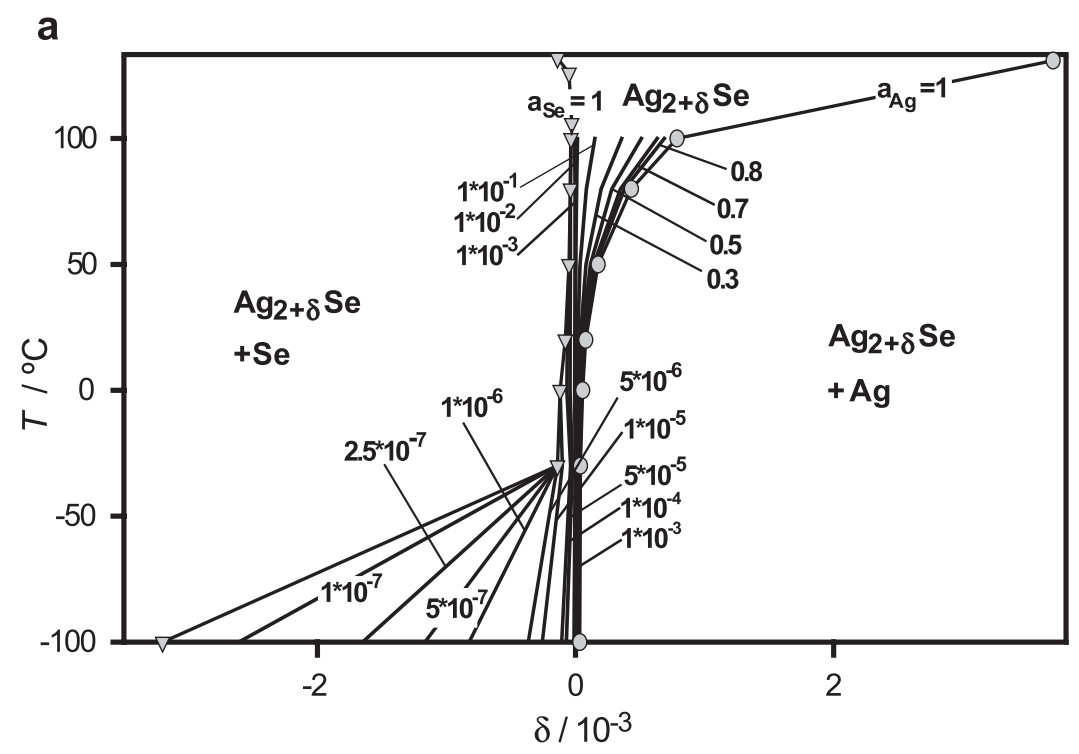

b

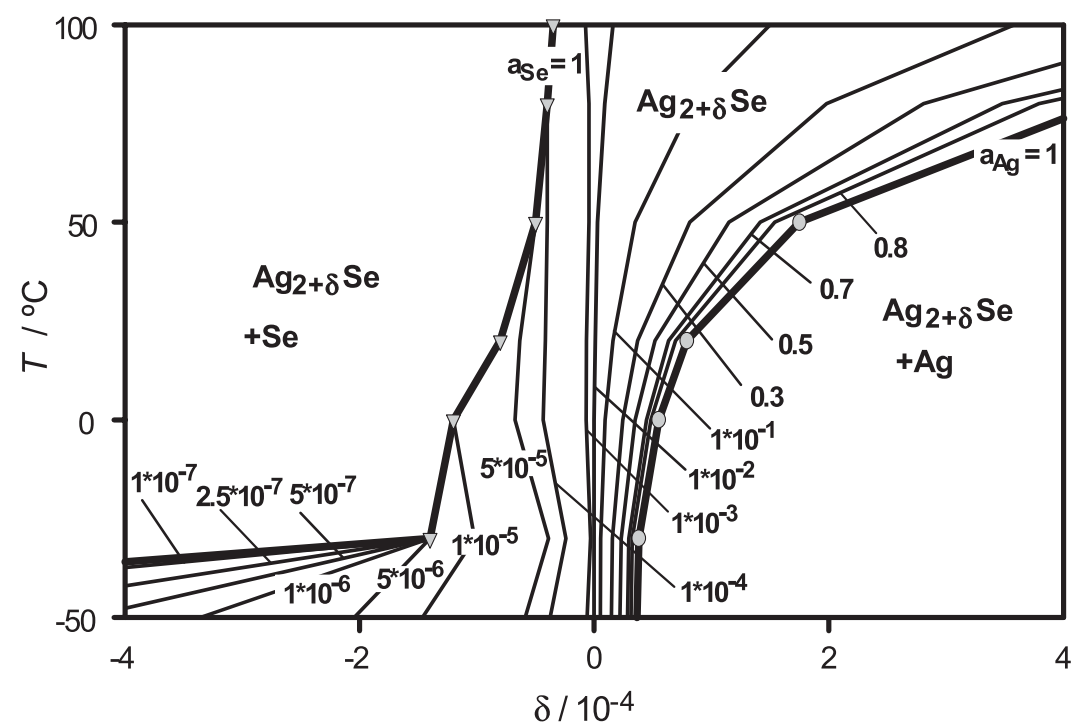

Fig. 3. (a) Phase field of $\alpha$-silver selenide down to $-100{ }^{\circ} \mathrm{C}$, determined by coulometric titration. The two boundaries correspond to the equilibrium with either silver metal $\left(a_{\mathrm{Ag}}=1\right)$ or selenium $\left(a_{\mathrm{Se}}=1\right)$. The lines within the phase field mark equal silver activities (isoactivity lines). (b) Enlarged section of the phase field in (a) down to $-50{ }^{\circ} \mathrm{C}$.

ature of a miscibility gap these parameters behave such that the phase field becomes wider with decreasing temperatures. To our knowledge, there exists no information on phase transformations or structural changes of either $\mathrm{Ag}, \mathrm{Ag}_{2} \mathrm{Se}$ or $\mathrm{Se}$ at these low temperatures. In effect, the phase boundary $\mathrm{Ag}_{2} \mathrm{Se} / \mathrm{Se}$ has to be regarded with some care and additional experiments are probably required to clarify this. The data for the silver-rich boundary can be regarded as reliable.

From the temperature dependence of $K_{\mathrm{F}}$ and $K_{\mathrm{e}}$, we calculate the standard formation enthalpy of Frenkel defects $\Delta H_{\mathrm{F}}=23 \mathrm{~kJ} \cdot \mathrm{mol}^{-1}$ and of electron-hole formation $\Delta H_{\mathrm{e}}=30$ $\mathrm{kJ} \cdot \mathrm{mol}^{-1}(0.3 \mathrm{eV})$ for the mean temperature of $35^{\circ} \mathrm{C}\left(K_{\mathrm{F}}\right.$ and $K_{\mathrm{e}}$ at $-100{ }^{\circ} \mathrm{C}$ were not considered in this calculation).

In Ref. [8], $\Delta H_{\mathrm{F}}$ was determined as $86.5 \mathrm{~kJ} \cdot \mathrm{mol}^{-1}$ for a mean temperature of $105{ }^{\circ} \mathrm{C}$. The comparison with our value implies that $\Delta H_{\mathrm{F}}$ decreases seriously with decreasing temperature. The standard enthalpy of electron-hole formation corresponds satisfactorily to the band gap of $\alpha$-silver selenide. Junod et al. [9] determined the band gap of $\alpha$-silver selenide at $20^{\circ} \mathrm{C}$ as $0.15 \mathrm{eV}$ and found that the gap increases with increasing temperature. This and the magnitude of our experimental values correspond to this prediction.

\section{Conclusions}

Since direct isothermal coulometric titration by ionic currents is virtually impossible at low temperatures where thermally activated ion motion is hindered, we construct the corresponding titration curves indirectly. In principle, our 
approach avoids long distance chemical diffusion in the sample at low temperatures, which is always required for homogenisation during conventional titration. The cooling procedure only requires local equilibration by point defect reactions, as long as we do not leave the homogeneous phase field. The corresponding processes have much shorter relaxation times than chemical (macroscopic) diffusion. Of course it is necessary that the electric resistance of the solid electrolyte is still low enough in order to allow reliable measurements of the electromotive force of the galvanic cell. It should be noted that the boundary of the phase field at its silver-rich side is easily identified experimentally, as the EMF of the galvanic cell approaches a value of $0 \mathrm{~V}$ for the case of equilibrium between the compound and silver metal. The boundary of the selenium-rich side is then accessible via a simple calculation, if we take a selenium activity of 1 as the criterion.

Via a modified titration technique, we were able to determine the nonstoichiometry of silver selenide at low temperatures. The boundary of the silver-rich side of the phase field has been constructed down to $-100{ }^{\circ} \mathrm{C}$. The homogeneous metal excess $\delta$ at this temperature equals $3.3 \times 10^{-5}$ and is much lower than the metal excess at the typical growth temperatures of silver selenide specimens ( $\delta=8 \times 10^{-3}$ at $\left.T=126{ }^{\circ} \mathrm{C}[7]\right)$. Thus, on cooling silver selenide from higher temperatures, virtually all silver metal excess has to be precipitated as a second phase in order to obtain equilibrium samples. Only very rapid cooling might prevent this precipitation at least partially [10]. As the chemical diffusion coefficient of silver metal in all silver chalcogenides is relatively high, we regard it as almost impossible to keep silver selenide specimens homogeneous during cooling. And thus, we have to conclude that the MR behaviour of silver selenide, which is usually investigated below room temperature, depends crucially not only on the growth but also on the cooling conditions. In a forthcoming study, we will report on the MR properties of silver selenide, which is carefully prepared and cooled such that indeed homogeneity is maintained.

\section{Acknowledgements}

This study is part of a project financed by the DFG (Ja648/5-1). Financial support by the FCI (Fonds der Chemischen Industrie) and critical reading of the manuscript by $\mathrm{C}$. Korte are also gratefully acknowledged.

\section{References}

[1] R. Xu, A. Husmann, T.F. Rosenbaum, M.-L. Saboungi, J.E. Enderby, B. Littlewood, Nature 390 (1997) 57.

[2] Z. Ogorelec, A. Hamzic, M. Basletic, Europhys. Lett. 46/1 (1999) 56.

[3] G. Beck, J. Janek, Phys., B Condens. Matter 308-310 (2001) 1086.

[4] U.v. Oehsen, H. Schmalzried, Ber. Bunsenges. Phys. Chem. 85 (1981) 7.

[5] N. Valverde, Z. Phys. Chem. (N.F.) 70 (1970) 128.

[6] C. Wagner, J. Chem. Phys. 21 (1953) 1816.

[7] W. Sitte, Ber. Bunsenges. Phys. Chem. 101 (9) (1997) 1372.

[8] C. Korte, J. Janek, Z. Phys. Chem. 206 (1998) 129.

[9] P. Junod, H. Hediger, B. Kilchör, J. Wullschleger, Philos. Mag. 6 (1977) 941.

[10] T. Ohachi, M. Hiramoto, Y. Yoshihara, I. Taniguchi, Solid State Ionics 51 (1992) 191. 